\title{
Lesiones accidentales en adultos mayores: un reto para los sistemas de salud
}

\author{
Ma. Guadalupe Ruelas González, Dr en C, ${ }^{(I)}$ V Nelly Salgado de Snyder, PhD. ${ }^{(I)}$
}

\section{Ruelas-González MG, Salgado de SnyderVN. Lesiones accidentales en adultos mayores: un reto para los sistemas de salud. Salud Publica Mex 2008;50:463-47I.}

Ruelas-González MG, Salgado de SnyderVN.

Accidental injuries in older adults: a challenge for the health systems. Salud Publica Mex 2008;50:463-47I.

\begin{abstract}
Resumen
Objetivo. Identificar los factores sociodemográficos, de salud y apoyo social relacionados con las lesiones accidentales en adultos mayores residentes de colonias urbanas marginales de las ciudades de Cuernavaca, Chilpancingo, Guadalajara y Culiacán. Material y métodos. En 2004-2005 se llevó a cabo un estudio transversal; mediante muestra no probabilística, se entrevistó a 799 adultos mayores; se emplearon pruebas no paramétricas y un modelo multivariado de regresión logística. Resultados. Del total de entrevistas, $37 \%$ notificaron lesiones y la causa principal fueron las caídas (54\%). El hogar fue el sitio con mayor número de accidentes (52\%). Los factores de riesgo asociados fueron edad avanzada, trabajar, mayor número de enfermedades, mayor consumo de medicamentos, remedios y bebidas alcohólicas, percepción de "mala salud", apoyo familiar inadecuado y ser cuidador de otros. Conclusiones. La multicausalidad obliga, por una parte, a que toda la sociedad participe y, por la otra, a la intervención del área de la salud para prevenir y atender el problema.
\end{abstract}

Palabras clave:adulto mayor; lesiones, accidentes; marginación; México

\begin{abstract}
Objective.To identify factors (sociodemographic, health, and social support) associated with the presence of accidental injuries in older adults living in deprived urban neighborhoods in four Mexican municipalities. Material and Methods. Cross-sectional survey carried out in 2004-2005, with a non-probabilistic, intentional sample of 799 male and female elderly living in deprived urban areas in four Mexican municipalities. For the statistical analysis, non-parametric tests and multivariate logistic regression models were used. Results. More than a third (37\%) of the sample reported injuries; with falls being the main cause. Home was the venue where most accidents took place (52\%). Risk factors for accidental injuries were: advanced age, working, greater number of illnesses, consumption of more medicines and remedies, self-perception of "poor" health , consumption of alcoholic beverages, inadequate family support and being a caretaker of others. Conclusions. The multi-causality of accidental injuries in older adults demands the participation of all sectors of society, and particularly public health interventions.
\end{abstract}

Key words: elderly; injuries; accidents; poverty; Mexico

(I) Centro de Investigación en Sistemas de Salud, Instituto Nacional de Salud Pública. Cuernavaca, Morelos, México.

Fecha de recibido: 29 de agosto de 2007 - Fecha de aceptado: 14 de mayo de 2008 Solicitud de sobretiros: Dra. V. Nelly Salgado de Snyder. Centro de Investigación en Sistemas de Salud, Instituto Nacional de Salud Pública. Av. Universidad 655, col Santa María Ahuacatitlán. 62508 Cuernavaca, Morelos, México. Correo electrónico:nsnyder@insp.mx 
$\mathrm{E}_{\mathrm{o}}^{\mathrm{n}}$ América Latina y el Caribe las lesiones traumáticas ocasionan $10 \%$ de la mortalidad y $18 \%$ de los años de vida potencialmente perdidos por discapacidad. ${ }^{1}$ Las lesiones son un problema de salud pública porque se encuentran vinculadas a la discapacidad, a la disminución de la calidad de vida y al incremento del número de años de vida potencialmente perdidos. ${ }^{2}$ Además, las lesiones demandan una mayor inversión de recursos económicos, sociales y humanos, la planeación de programas especializados y la redefinición de prioridades en salud.

En México, como en muchos países en desarrollo, los retos de las transiciones epidemiológica ${ }^{3}$ y demográfica han contribuido a que las lesiones en el adulto mayor (AM) sean reconocidas como un importante problema de salud pública ${ }^{4}$ por su aportación a la carga de mortalidad y morbilidad y por los altos costos para los sistemas de salud. ${ }^{5,6} \mathrm{El}$ rápido aumento de la población de $\mathrm{AM}, 7,8$ la insuficiente cobertura de los sistemas de seguridad social, ${ }^{5}$ así como una probable tendencia hacia la disminución del apoyo - producto de los cambios en la estructura y composición familiar-9-11 determinan que las lesiones en los AM sean un tema de prioridad nacional.

La información sobre lesiones en el AM es limitada, dispersa y no se encuentra sistematizada; por esta razón, se desconoce la magnitud de las lesiones accidentales y problemas asociados en los AM del país. Sin embargo, existen algunos indicadores de esta problemática: por ejemplo, en 2003 los accidentes en México ocuparon el $8^{\circ}$ lugar entre las principales causas de mortalidad en el grupo de 65 años y más. ${ }^{12,13}$ Las dos causas más importantes de defunción por lesiones en los AM fueron los accidentes de tránsito (primera causa específica de muerte) y las caídas (primera causa de mortalidad hospitalaria). En el mismo año, la mortalidad hospitalaria por lesiones en personas de 60 años y más fue de 35.7\% y la morbilidad de $14.9 \%{ }^{14}$

Las complicaciones derivadas de las lesiones ejercen un impacto adverso sobre la red social y la economía del anciano y de su hogar, así como sobre la sociedad en general y los sistemas de salud, en particular. ${ }^{1}$ Por ejemplo, las consecuencias de las lesiones en los AM pueden variar desde un hematoma hasta una fractura e, inclusive, la muerte, en tanto que en el plano psicológico pueden ocurrir cambios en el comportamiento y actitudes que se traducen en una mayor dependencia. Finalmente, se ha documentado que la susceptibilidad de los ancianos a las complicaciones por lesiones está relacionada con la alta prevalencia de enfermedades concomitantes y cambios que suceden durante el proceso de envejecimiento. ${ }^{15}$
El objetivo de la presente contribución fue identificar algunos factores (sociales, demográficos y de salud) relacionados con la notificación de lesiones accidentales en AM residentes de zonas urbanas marginadas de cuatro municipios de México.

\section{Material y métodos}

Se realizó un estudio transversal con base comunitaria, entre junio de 2004 y agosto de 2005, con una muestra intencional, no probabilística, de 799 AM de ambos sexos (60 años y más) residentes de colonias urbano marginales de los municipios de Cuernavaca, Chilpancingo, Guadalajara y Culiacán. ${ }^{*}$ Con respecto al poder estadístico del estudio, se obtuvo una prevalencia de $37 \%$, con una precisión de $\pm 2 \%$ y un nivel de confianza de $95 \%$, con poder superior a $90 \%$.

Los AM participantes se reclutaron utilizando listados de los dirigentes de las colonias, centros de salud, iglesias y referencias comunales. Tanto las autoridades municipales respectivas como el Consejo Estatal de Población identificaron a las colonias como de "muy alta" y "alta" marginación, con base en los criterios establecidos por el Consejo Nacional de Población (CONAPO). *,16 Antes de la administración de la encuesta se aplicó una versión breve y modificada del Mini-mental-test de Folstein, ${ }^{17}$ mediante el cual se evaluó únicamente la orientación en tiempo, espacio y persona. Dado que el estudio se basó en auto reporte, no se encuestó a AM con disfunción cognitiva, del habla o del lenguaje, ni a aquellos que estaban institucionalizados. Asimismo, a los participantes se les explicó el objetivo del estudio, se aseguró la confidencialidad y anonimato en sus respuestas y se les solicitó firmar su consentimiento informado de acuerdo con lo establecido en los principios éticos para las investigaciones con sujetos humanos de la Declaración de Helsinki.

Para la recolección de datos se requirió de un cuestionario utilizado en un estudio anterior ${ }^{18}$ con AM rurales. El instrumento fue administrado por encuestadores con licenciatura quienes, además, tomaron un curso de 40 horas sobre su manejo y aplicación, el cual incorporó un total de 11 secciones. En este trabajo las cuatro secciones del cuestionario que se analizaron fueron: a) características sociodemográficas: sexo, edad, escolaridad, estado civil, hijos y situación laboral; b) estado de salud a través de auto reporte: cuáles y cuántas

\footnotetext{
* Investigación que se deriva del proyecto financiado por el Consejo Nacional de Ciencia y Tecnología "Envejecimiento, pobreza y salud en población urbana" (Proyecto CONACYT 2002-CO1-7419).
} 
enfermedades padecieron en el año previo a la encuesta; remedios y medicamentos consumidos; percepción de su estado de salud; consumo de alcohol y puntaje del CES-D para evaluar sintomatología depresiva; ${ }^{19}$ c) apoyo social: número de horas al día que permanecía sólo, presencia de apoyo instrumental y emocional de personas que no vivían con ellos; si era cuidador de otra persona, si contaba con algún apoyo económico y si realizaba labores en el hogar; d) apoyo familiar: escala de 13 reactivos, cuyo punto de corte fue la media más una desviación estándar como indicador de apoyo familiar "inadecuado".

Las anotaciones del encuestador se hacían inmediatamente después de la entrevista y requerían señalar si el anciano mostraba lesiones evidentes y si en la vivienda había riesgos físicos de lesionarse. A través del auto reporte se determinó la variable dependiente, preguntando directamente al anciano si en el último año había sufrido alguna lesión (herida, contusión, quemadura, luxación, fractura, otros). El ponderado final fue (0) para aquellos que no notificaron lesión y (1) para los que sí lo hicieron.

Para describir las lesiones se identificaron los tipos y la región anatómica afectada y se agruparon por causa externa, codificándolos de acuerdo con la Clasificación Internacional de Enfermedades (CIE-10) (V01-X59), de tal manera que quedaron cinco grupos. En los primeros cuatro, los lesionados informaron sólo una de las siguientes causas: 1) fuerzas mecánicas inanimadas; 2) caídas; 3) fuego y sustancias calientes, y 4) otras causas externas. En este último grupo se incluyeron todas las causas que tuvieron baja frecuencia como accidentes de tránsito, accidentes ocasionados por animales, exceso de esfuerzo y factores no especificados. En el grupo 5, "diversas causas externas", se clasificaron lesiones ocasionada por dos o más causas externas, por ejemplo una herida por cuchillo al preparar los alimentos y una luxación por caerse al tropezar con algún objeto. Las causas externas se categorizaron por el lugar donde ocurrió la lesión, número de lesiones y tipo de consecuencias.

\section{Análisis de datos}

En el análisis bivariado para explorar la relación entre lesionados y no lesionados, se utilizaron las pruebas de ji cuadrada y Mann-Withney. De igual forma se empleó la regresión logística binaria simple y se calcularon razones de momios $(R M)$ con intervalos de confianza a 95\% (IC 95\%). En el modelo multivariado final se incluyeron las variables que en el análisis bivariado tuvieron un valor de $\leq 0.25$. Se realizó el análisis del factor de inflación de la varianza (VIF) con el propósito de evaluar la multicolinealidad entre las variables independientes. Se probaron interacciones y permanecieron en el modelo cuando su valor $p$ era menor a 0.15 . Se empleó la prueba de error de especificación (linktest) para probar el supuesto que asume que el logit de la variable de respuesta es una combinación lineal de las variables independientes. El ajuste del modelo se llevó a cabo con la prueba de bondad de ajuste, usando un punto de corte de $p>0.10$ para considerar un ajuste adecuado. ${ }^{20}$ Para el procesamiento de la información se utilizó el paquete estadístico Stata, versión 9.

\section{Resultados}

La muestra constó de un total de 799 AM, de los cuales 394 eran varones (49\%) y 405 mujeres (51\%), con una mediana de 68 años de edad. De ellos, 56\% tenían pareja, $94 \%$ informaron tener hijos, 55\% no recibían apoyo económico alguno y $39 \%$ trabajaban en oficios diversos (albañil, plomero, pintor, jardinero, quehaceres domésticos y comercio informal, entre otros).

Del total de la muestra, 37\% $(\mathrm{n}=290)$ notificaron haber sufrido alguna lesión el año anterior a la encuesta. En el cuadro I se presentan los resultados descriptivos, contrastando las características de los que presentaron y no presentaron lesiones. Cabe destacar que el grupo de mujeres tuvo la mayor proporción de lesionados; asimismo, entre lesionados y no lesionados se encontraron diferencias $(p<0.05)$; los lesionados informaron padecer un mayor número de enfermedades, y también entre ellos se encontró la proporción más alta de consumidores de más de un remedio casero. Si bien el consumo de medicamentos no fue significativo, es importante mencionar que sólo uno de cada cuatro AM tomaba medicamentos bajo prescripción médica; el resto se automedicaba. De igual forma, entre los lesionados se encontró la mayor proporción con sintomatología depresiva y que consumía alcohol, principalmente cerveza, con una periodicidad de "diario" o "tres veces por mes". Finalmente, aunque una mayor proporción de lesionados manifestó la ausencia de apoyo familiar adecuado, informaron contar con apoyo social emocional procedente de personas que no vivían con ellos; asimismo, en este grupo se encontró la mayor proporción de cuidadores de otros.

El número total de lesiones notificadas por los participantes en este estudio fue de 366, distribuidas de la siguiente manera: 224 AM señalaron una lesión; 58 indicaron dos lesiones, y ocho manifestaron haber tenido tres o más lesiones en el último año.

Como se observa en los cuadros II y III, las partes del cuerpo más afectadas fueron las extremidades superiores e inferiores. Los hombres informaron 159 lesiones ocasionadas, sobre todo, por fuerzas mecánicas inanima- 
Cuadro I

Características de los adultos mayores de acuerdo con el informe de lesión

\begin{tabular}{|c|c|c|c|c|c|}
\hline \multirow[b]{2}{*}{ Variables } & \multicolumn{2}{|c|}{ No lesionados } & \multicolumn{2}{|c|}{ Lesionados } & \multirow[t]{2}{*}{$p$} \\
\hline & $n=509$ & (\%) & $n=290$ & (\%) & \\
\hline \multicolumn{6}{|l|}{ Sexo } \\
\hline Hombre & 263 & 51.67 & $|3|$ & 45.17 & \\
\hline Mujer & 246 & 48.33 & 159 & 54.83 & 0.07 \\
\hline Edad* & Med 68 & RIC 63-74 & Med 69 & RIC 64-75 & 0.28 \\
\hline Años de escuela cursados* & Med 0 & RIC $0-2$ & Med 0 & RIC 0-3 & 0.10 \\
\hline \multicolumn{6}{|l|}{ Tiene compañero/a } \\
\hline Sí & 278 & 54.62 & 169 & 58.28 & \\
\hline No & 231 & 45.38 & 121 & 41.72 & 0.36 \\
\hline \multicolumn{6}{|l|}{ Hijos } \\
\hline Sí & 485 & 95.28 & 268 & 92.41 & 0.09 \\
\hline \multicolumn{6}{|l|}{ Trabaja } \\
\hline Sí & 195 & 38.31 & 117 & 40.34 & 0.57 \\
\hline \multicolumn{6}{|c|}{ Tiene ingresos o apoyo económico } \\
\hline No & 275 & 54.03 & 165 & 56.90 & 0.43 \\
\hline Número enfermedades $*^{\ddagger}$ & Med 3 & RIC 2-5 & Med 4 & RIC 2-6 & 0.00 \\
\hline \multicolumn{6}{|l|}{ Consumo remedios } \\
\hline No consumo & 275 & 54.03 & $|3|$ & 45.17 & \\
\hline $1-3$ & 215 & 42.24 & 133 & 45.86 & \\
\hline$>4$ & 19 & 3.73 & 26 & 8.97 & 0.00 \\
\hline \multicolumn{6}{|l|}{ Consumo medicamentos } \\
\hline No consumo & 199 & 39.10 & 100 & 34.48 & \\
\hline $1-3$ & 283 & 55.60 & 165 & 56.90 & \\
\hline$>4$ & 27 & 5.30 & 25 & 8.62 & 0.12 \\
\hline \multicolumn{6}{|l|}{ Sintomatología depresiva } \\
\hline Sí & 78 & 15.32 & 65 & 22.41 & 0.01 \\
\hline \multicolumn{6}{|l|}{ Percepción estado salud } \\
\hline Bueno - excelente & 247 & 50.61 & 127 & $44.4 I$ & \\
\hline Regular & 198 & 40.57 & 114 & 39.86 & \\
\hline Malo - muy malo & 43 & 8.81 & 45 & 15.73 & 0.24 \\
\hline \multicolumn{6}{|l|}{ Consumo alcohol } \\
\hline No consumo & 353 & 69.35 & 190 & 65.52 & \\
\hline |-2 copas por ocasión & 120 & 23.58 & 70 & 24.14 & \\
\hline$\geq 3$ copas por ocasión & 36 & 7.07 & 30 & 10.34 & 0.04 \\
\hline \multicolumn{6}{|l|}{ Apoyo familiar } \\
\hline Inadecuado & 91 & 17.88 & 72 & 24.830 & 0.01 \\
\hline \multicolumn{6}{|l|}{ Apoyo emocional social } \\
\hline Sí & 152 & 29.86 & 107 & 36.90 & 0.04 \\
\hline \multicolumn{6}{|l|}{ Apoyo instrumental social } \\
\hline Sí & 244 & 47.94 & 158 & 54.48 & 0.07 \\
\hline \multicolumn{6}{|l|}{ Cuidador de otro } \\
\hline Sí & 161 & 31.63 & 114 & 39.31 & 0.02 \\
\hline \multicolumn{6}{|l|}{ Labores en el hogar } \\
\hline Sí & 265 & 52.06 & 160 & 55.17 & 0.39 \\
\hline Número horas al día solo* & Med 2 & RIC 0-8 & Med 3 & RIC 0-8 & 0.14 \\
\hline \multicolumn{6}{|l|}{ Piso de tierra en casa } \\
\hline Sí & 138 & 34.76 & 96 & 40.00 & 0.18 \\
\hline Techo de lámina, asbesto o & & & & & \\
\hline Sí & 180 & 44.89 & 114 & 47.30 & 0.55 \\
\hline Mascotas en casa & & & & & \\
\hline Sí & 179 & 44.53 & 116 & 49.79 & 0.20 \\
\hline
\end{tabular}

* Mediana, rango intercuartil $25-75$. Prueba de Mann-Withney

‡ Por autorreporte

Fuente: encuesta efectuada en colonias urbanas marginales de Cuernavaca, Chilpancingo, Guadalajara y Culiacán, entre junio 2004 y agosto 2005, para el proyecto de investigación "Envejecimiento, pobreza y salud en población urbana" 


\section{Cuadro II}

TIPO DE LESIONES EN ADULTOS MAYORES NOTIFICADAS Y PARTE DEL CUERPO AFECTADA

\begin{tabular}{lcccccccr} 
Tipo Lesión & Heridas & Contusiones & Quemaduras & Luxaciones & Fracturas & Total & (\%) \\
Cabeza & 10 & 47 & 1 & 4 & 3 & 64 & $(17.5)$ \\
\hline Tórax & 5 & 9 & 2 & 8 & 2 & 27 & $(7.5)$ \\
\hline Cadera & 3 & 11 & - & 7 & 7 & 28 & $(7.5)$ \\
\hline Extremidades inferiores & 25 & 37 & 5 & 31 & 10 & 108 & $(29.5)$ \\
\hline Extremidades superiores & 49 & 20 & 36 & 18 & 16 & 139 & $(38.0)$ \\
\hline Total & 92 & 124 & 44 & 68 & 38 & 366 & $(100 \%)$
\end{tabular}

Fuente: encuesta efectuada en colonias urbanas marginales de Cuernavaca, Chilpancingo, Guadalajara y Culiacán, entre junio 2004 y agosto 2005, para el proyecto de investigación "Envejecimiento, pobreza y salud en población urbana"

\section{Cuadro III}

\section{Causas externas de lesiones en AdULtos mayores por lugar de ocurrencia}

\begin{tabular}{lccccccc} 
Lugar & Causas externas & Total (\%) & FMF & Caidas & FSC & Otras causas externas & Diferentes $^{\circledR}$ causas externas $^{\ddagger}$ \\
Hogar & 29 & 84 & 21 & 6 & 13 & 153 & $(52.16)$ \\
\hline Vía pública & 8 & 47 & 2 & 7 & 2 & 66 & $(22.76)$ \\
\hline Trabajo & 20 & 21 & 2 & 4 & 3 & 50 & $(17.24)$ \\
\hline Varios & 1 & 4 & - & - & 16 & 21 & $(7.24)$ \\
\hline Total & 58 & 156 & 25 & 17 & 34 & 290 & $(100 \%)$
\end{tabular}

Códigos de Clasificación Internacional de Enfermedades (CIE) $10^{a}$ revisión:

* Fuerzas mecánicas inanimadas (W20,22,25-27,45)

\# Caídas (W0I,03,05-13,17-19)

$\S$ Fuego y sustancias calientes (X02,10-12)

\# Otras causas externas

\& Diversas causas externas (dos o más causas externas)

Fuente: encuesta efectuada en colonias urbanas marginales de Cuernavaca, Chilpancingo, Guadalajara y Culiacán, entre junio 2004 y agosto 2005, para el proyecto de investigación "Envejecimiento, pobreza y salud en población urbana"

das, en tanto que las mujeres notificaron 207 debidas en su mayoría a caídas, fuego y sustancias calientes. Cabe destacar que los varones sufrieron de heridas y fracturas con mayor frecuencia que las mujeres, en tanto que estas últimas tuvieron más contusiones, quemaduras y luxaciones que los hombres.

En el cuadro III se describen las lesiones desglosadas por causa externa; hubo 58 (20\%) atribuidas a fuerzas mecánicas inanimadas: golpes, contra o golpeado por objetos que caen; uso de objetos punzo-cortantes; cuerpo extraño que penetra a través de la piel, y contacto traumático con herramientas manuales.

Las caídas (resbalón, tropezón o traspié y por caídas de la cama, silla, escalera o banqueta) fueron la principal causa de lesión en los AM; en total se notificaron 156 $(54 \%)$. En general los participantes señalaron que las caí- das se debieron principalmente a objetos mal ubicados o en mal estado: palos, piedras, trastos, sillas o al bajar alguna banqueta. Resulta pertinente indicar que 36\% de los hombres que se lesionaron como consecuencia de una caída trabajaban como albañiles.

Veinticinco AM $(8 \%)$ señalaron lesiones por fuego y sustancias calientes (agua, aceite, alimentos, estufa o brasas). En "otras causas externas" se agruparon aquellas que tuvieron baja frecuencia como: accidentes de transporte, mordidas de animal y piquetes de aves; $17 \mathrm{AM}(6 \%)$ indicaron este tipo de lesiones. Finalmente 34 AM $(12 \%)$ señalaron que en el último año sufrieron lesiones ocasionadas por más de una causa externa.

En el cuadro III se detallan, asimismo, las causas externas de las lesiones por lugar de ocurrencia. El hogar fue el sitio donde se presentó el mayor número 
de hechos accidentales (52\%). Las mujeres notificaron una mayor frecuencia de accidentes en el hogar $(n=104)$ y los hombres en la vía pública $(\mathrm{n}=35)$ y en el trabajo $(\mathrm{n}=41)$.

En cuanto a las consecuencias de las lesiones, en total $125(43 \%)$ participantes (mujeres 72 , hombres 53 ) indicaron haber tenido consecuencias negativas por las lesiones sufridas. Las que se notificaron con mayor frecuencia fueron "nervios" y "dolor" $(\mathrm{n}=41)$, siendo más afectadas las mujeres que los hombres. De igual manera se mencionaron limitaciones o inmovilidad $(\mathrm{n}=35)$ en la parte del cuerpo afectada, además de otras consecuencias como "enfermedad" y "debilidad".

Cabe señalar que 71 entrevistados (24\%) presentaban lesiones evidentes al momento de aplicar la encuesta, pero sólo cinco lo informaron verbalmente. De los AM lesionados 16\% ( $\mathrm{n}=130)$ manifestaron esta situación como consecuencia de maltrato asociado a conflictos familiares. Los resultados del análisis de maltrato y lesiones se describen en otro trabajo, ${ }^{21}$ ya que el presente se enfocó exclusivamente a las lesiones accidentales.

Los resultados del análisis bivariado sugieren que los hombres lesionados, comparados con los no lesionados $(p<0.05)$, tendieron a estar casados, tener mayor escolaridad, poseer mascotas, no contar con apoyo familiar adecuado, ser cuidador de otros y consumir un mayor número de remedios. En cuanto a las mujeres que notificaron lesiones, al compararlas con las que no lo hicieron $(p<0.05)$, fueron las de mayor edad, no contaban con apoyo social adecuado, pasaban solas un mayor número de horas al día y la percepción de su estado de salud era de "malo a muy malo". Para ambos sexos también resultó significativo haber padecido un mayor número de enfermedades.

En el cuadro IV se observan los resultados del análisis bivariado en los AM lesionados. Las variables edad, tener pareja y trabajar, obtuvieron una $p>0.25$; sin embargo, al ser integradas en el modelo multivariado resultaron significativas.

En el cuadro V se presenta el modelo multivariado, que consta de once efectos principales y una interacción entre el ser mujer y tener compañero. Se estimó que la posibilidad de presentar lesiones aumenta conforme avanza la edad, el número de enfermedades, el consumo de más de cuatro medicamentos y más de cuatro remedios. Asimismo, la posibilidad de tener lesiones aumenta entre los que trabajan, los que perciben tener mala salud, los que consumen más de tres bebidas alcohólicas por ocasión, los que no cuentan con apoyo familiar inadecuado y los que son cuidadores de otros. Por otra parte, como se ilustra en la parte inferior del

\begin{tabular}{|c|c|c|c|}
\hline $\begin{array}{r}\text { VARIABLES REL } \\
\text { ACCIDENTA }\end{array}$ & $\begin{array}{l}\text { dro IV } \\
\text { ADAS CON } \\
\text { EL ADULT }\end{array}$ & $\begin{array}{l}\text { AS LESIONES } \\
\text { MAYOR }\end{array}$ & \\
\hline Variables & RM cruda & (IC 95\%) & $p$ \\
\hline Número enfermedades $^{\ddagger}$ & 1.13 & $(1.07-1.20)$ & 0.000 \\
\hline \multicolumn{4}{|l|}{ Consumo medicamentos } \\
\hline No consumo & & $I^{*}$ & \\
\hline $1-3$ & 1.16 & $(0.85-I .57)$ & 0.343 \\
\hline$>4$ & 1.84 & $(1.01-3.33)$ & 0.044 \\
\hline \multicolumn{4}{|l|}{ Consumo remedios } \\
\hline No consumo & & $I^{*}$ & \\
\hline $\mid-3$ & 1.29 & $(0.96-1.75)$ & 0.088 \\
\hline$>4$ & 2.87 & $(1.53-5.37)$ & 0.001 \\
\hline \multicolumn{4}{|l|}{ Percepción estado salud } \\
\hline Bueno - excelente & & $I^{*}$ & \\
\hline Regular & I.II & $(0.81-1.53)$ & 0.481 \\
\hline Malo - muy malo & 2.03 & $(1.27-3.25)$ & 0.003 \\
\hline \multicolumn{4}{|c|}{ Presentar sintomatología depresiva } \\
\hline No & & $I^{*}$ & \\
\hline Sí & 1.56 & $(1.10-2.30)$ & 0.012 \\
\hline \multicolumn{4}{|l|}{ Apoyo familiar } \\
\hline Adecuado & & $I^{*}$ & \\
\hline Inadecuado & 1.51 & $(1.06-2.15)$ & 0.020 \\
\hline \multicolumn{4}{|l|}{ Sexo } \\
\hline Hombres & & $I^{*}$ & \\
\hline Mujeres & 1.29 & $(0.97-1.73)$ & 0.078 \\
\hline \multicolumn{4}{|l|}{ Consumo alcohol } \\
\hline No consumo & & $I^{*}$ & \\
\hline I-2 copas por ocasión & 1.08 & $(0.76-1.52)$ & 0.646 \\
\hline$\geq 3$ copas por ocasión & 1.54 & $(0.92-2.59)$ & 0.097 \\
\hline \multicolumn{4}{|l|}{ Cuidador de otro } \\
\hline No & & $I^{*}$ & \\
\hline Sí & 1.30 & $(0.97-1.73)$ & 0.078 \\
\hline Edad & 1.00 & $(0.99-1.02)$ & 0.726 \\
\hline \multicolumn{4}{|l|}{ Tiene compañero/a } \\
\hline No & & $I^{*}$ & \\
\hline Sí & 1.16 & $(0.86-1.55)$ & 0.317 \\
\hline \multicolumn{4}{|l|}{ Trabaja } \\
\hline No & & $I^{*}$ & \\
\hline Sí & 1.08 & $(0.81-1.46)$ & 0.571 \\
\hline
\end{tabular}

Fuente: encuesta efectuada en colonias urbanas marginales de Cuernavaca, Chilpancingo, Guadalajara y Culiacán, entre junio 2004 y agosto 2005 para el proyecto de investigación "Envejecimiento, pobreza y salud en población urbana" 


\section{Cuadro V}

ANÁLISIS MULTIVARIADO DE REGRESIÓN LOGíSTICA DE ADULTOS MAYORES LESIONADOS

\begin{tabular}{lccc} 
Variables & RM ajustada & (IC 95\%) & $P$ \\
Edad & 1.02 & $(0.99-1.04)$ & 0.056 \\
\hline $\begin{array}{l}\text { Trabaja } \\
\text { No }\end{array}$ & & $I^{*}$ & \\
$\quad$ Sí & 1.38 & $(0.98-1.94)$ & 0.064 \\
\hline Número enfermedades & 1.10 & $(1.03-1.16)$ & 0.001 \\
\hline $\begin{array}{l}\text { Consumo medicamentos } \\
\text { 0-3 }\end{array}$ & & & \\
$>4$ & 2.99 & $(1.24-7.20)$ & 0.014 \\
\hline
\end{tabular}

\begin{tabular}{|c|c|c|c|}
\hline \multicolumn{4}{|l|}{ Consumo remedios } \\
\hline $0-3$ & & $I^{*}$ & \\
\hline$>4$ & 2.05 & $(1.07-3.91)$ & 0.029 \\
\hline \multicolumn{4}{|l|}{ Percepción estado salud } \\
\hline Bueno - regular & & $I^{*}$ & \\
\hline Malo - muy malo & 1.70 & $(1.05-2.76)$ & 0.031 \\
\hline \multicolumn{4}{|l|}{ Consumo alcohol } \\
\hline $0-2$ copas por ocasión & & $I^{*}$ & \\
\hline$\geq 3$ copas por ocasión & 1.81 & $(1.03-3.19)$ & 0.037 \\
\hline \multicolumn{4}{|l|}{ Apoyo familiar } \\
\hline Adecuado & & $I^{*}$ & \\
\hline Inadecuado & 1.49 & $(1.03-2.17)$ & 0.033 \\
\hline \multicolumn{4}{|l|}{ Cuidador de otro } \\
\hline No & & $I^{*}$ & \\
\hline Sí & 1.40 & $(1.01-1.93)$ & 0.041 \\
\hline \multicolumn{4}{|l|}{ Sexo } \\
\hline Hombres & & $I^{*}$ & \\
\hline Mujeres & $\ddagger 2.33$ & $(1.36-3.99)$ & 0.002 \\
\hline \multicolumn{4}{|l|}{ Tiene compañero/a } \\
\hline No & & $I^{*}$ & \\
\hline Sí & $\ddagger 1.94$ & $(1.15-3.27)$ & 0.012 \\
\hline Mujer/hombre con compañero(a) & $\neq 0.524$ & $(0.26-1.02)$ & 0.057 \\
\hline Mujer sin compañero & 2.33 & $(1.36-4.00$ & 0.002 \\
\hline Mujer con compañero & 1.22 & $(0.78-1.91)$ & 0.357 \\
\hline
\end{tabular}

* Referencia

‡ Interacción entre sexo y tener compañero

Fuente: encuesta efectuada en colonias urbanas marginales de Cuernavaca, Chilpancingo, Guadalajara y Culiacán, entre junio 2004 y agosto 2005 para el proyecto de investigación "Envejecimiento, pobreza y salud en población urbana"

Prueba de bondad de ajuste Ifit, group (I0): number of observations= 799; Hosmer-Lemeshow chi2(8) $=5.75 ;$ Prob $>$ chi2 $=0.6755$. Prueba de error de especificación (linktest) hat $p=0.0000$; hatsq $p=0.970$ cuadro $\mathrm{V}$, las mujeres tienen un momio de lesionarse 2.33 veces más en comparación con los hombres, y el tener compañero aumenta los momios $94 \%$ (IC=1.15-3.27) en relación con las que no tienen pareja.

La interacción que resultó estadísticamente significativa se puede explicar en términos de: 1) el efecto estimado de ser mujer en el grupo "sin pareja" $(R M=2.33 ; I C=1.36-4.00 ; p<0.002)$, y 2$)$ el efecto de ser mujer en el grupo "con pareja" $(R M=1.22 ; I C=0.78-1.91$; $p>0.370$ ). En otras palabras, la posibilidad de presentar lesiones fue mayor en las mujeres que no tenían compañero en relación con los hombres que no tenían compañera $(p<0.05)$. Sin embargo, esa posibilidad fue la misma para ambos sexos cuando notificaron tener pareja $(p>0.05)$.

\section{Discusión}

Los resultados de este estudio sugieren que, entre los factores de riesgo de lesión en el grupo estudiado se encuentran la edad avanzada, trabajar, padecer un mayor número de enfermedades, hacer un mayor consumo de medicamentos y remedios, la percepción de "mala" salud, el consumo de más de tres copas de alcohol por ocasión, apoyo familiar inadecuado, y ser cuidador de otros, así como la interacción de ser mujer y no tener compañero, todo lo cual muestra consistencia con lo notificado en estudios previos. ${ }^{22-26}$

Los AM participantes vivían en contextos de marginación, de tal manera que es posible que otros factores como la vivienda inadecuada, los bajos ingresos, el bajo nivel de educación y la falta de servicios adecuados también hayan contribuido a la presencia de lesiones. Asimismo, es factible que en algunos de los participantes la acumulación de síntomas infraclínicos, en ocasiones inadvertidos, expliquen parcialmente los accidentes que pueden ocasionar lesiones. ${ }^{27-28}$ Este estudio confirma hallazgos previos sobre la asociación entre el consumo de bebidas alcohólicas y lesiones en el AM. ${ }^{29-30}$ El consumo de alcohol es un riesgo potencial, especialmente cuando la ingesta se combina con polifarmacia, lo cual aumenta la posibilidad de presentar lesiones, sobre todo debido a caídas. ${ }^{31}$

Por otro lado, el apoyo familiar inadecuado se encontró asociado con la presencia de lesiones, mientras que la interacción de ser mujer y vivir con su pareja resultó ser un factor de protección para las lesiones. Estos resultados destacan la importancia del apoyo familiar para la promoción del bienestar generalizado en los AM. ${ }^{32}$ 
En otros países también se han documentado hallazgos sobre el papel fundamental del apoyo familiar en el restablecimiento de la salud de AM lesionados. ${ }^{33,34}$

El hogar fue el sitio donde se notificó la ocurrencia de la mayoría de las lesiones, lo cual concuerda con hallazgos previos. ${ }^{34}$ El INEGI ha notificado que el hogar ocupa el segundo lugar en frecuencia de defunciones por lesiones accidentales y violentas. ${ }^{11}$ Este dato enfatiza la importancia de identificar los factores de riesgo en los hogares de AM, particularmente los de aquellos que viven en pobreza, en viviendas de construcción precaria, ya que los AM tienden a pasar la mayor parte de su tiempo en el hogar. ${ }^{33}$

Una de las limitaciones de este trabajo es su diseño transversal, al no poder obtener relaciones de tipo causal entre las variables dependiente y las independientes. Por otro lado, la muestra no fue aleatoria y sus resultados no pueden ser extrapolados a la población general. Finalmente, el autorreporte y el sesgo de memoria podrían verse como limitaciones del estudio, sin embargo, el auto reporte ha sido reconocido como una medición costo-efectiva para estudios poblacionale ${ }^{35} \mathrm{y}$ los indicadores subjetivos, a pesar del posible sesgo de memoria, se han considerado como indicadores útiles en estudios con $\mathrm{AM}^{36}$

Las lesiones accidentales en los AM, sus causas y consecuencias, son temas que sólo hasta fechas muy recientes se han abordado en proyectos de investigación, por lo que es necesario fortalecer esta línea a través de estudios que midan la magnitud y distribución de las lesiones accidentales en AM que viven en diferentes contextos sociales. De la misma manera, en el área de lesiones es necesario identificar y entender el comportamiento de los factores de riesgo y los factores protectores en la vida cotidiana de los AM, tales como el papel de las redes y el apoyo social, emocional e instrumental que recibe y que proporciona el anciano a los miembros de su red social y familiar. Estas investigaciones deberán tener una perspectiva de género, ya que la participación de las AM en labores del hogar y como cuidadoras de otros $^{37}$ es poco valorada, ${ }^{11}$ provoca estrés, sobrecarga de trabajo y riesgos adicionales. ${ }^{26}$ Por ejemplo, en este estudio el manejo de fuego y sustancias calientes al cocinar fueron causa importante de lesiones entre las mujeres. Por otra parte, también se encontró una asociación significativa entre ser cuidadores de otros y la presencia de lesiones.

Uno de los retos más importantes para los sistemas de salud consiste en diseñar programas y acciones enfocados a prevenir las lesiones y sus consecuencias discapacitantes, sin descuidar la atención a las enfermedades crónicas que prevalecen entre los AM; ${ }^{38}$ además de la promoción de la salud a través de programas de auto-cuidados dirigidos a los AM, así como intervenciones para sensibilizar a la red familiar y a los prestadores de servicios sobre los riesgos físicos, biológicos y sociales asociados a las lesiones accidentales en los AM. Es esencial crear programas de capacitación para proveedores de servicios encaminados a la detección oportuna de lesiones y su tratamiento adecuado. Por último, el abordaje del problema de lesiones en el AM demanda acciones coordinadas, atención integral y la participación de todos los sectores de la sociedad, incluyendo a los propios AM y sus familiares, en el diseño de programas de prevención de lesiones accidentales.

\section{Reconocimientos}

Este estudio se realizó con recursos del Fondo Sectorial CONACYT-SALUD (C01-7419).

\section{Referencias}

I. Organización Panamericana de la Salud. La actividad de la OPS en el campo de las enfermedades no transmisibles. Rev Panam Salud Publica 1997;2:478-490.

2. Peden M, McGee K, Krug E. Injury: a leading cause of the global burden of disease. Washington, DC: World Health Organization 2002:2-26.

3. Lozano R. El peso de la enfermedad en México: avances y desafíos. En: Frenk J. Observatorio de la salud: necesidades y servicios, políticas. México: FUNSALUD, 1997: 23-61.

4. Secretaría de Salud. Programa de Acción:Accidentes 200I -2006. México: SSA, 2002.

5. Comisión Económica para América Latina y el Caribe. Las personas mayores en América Latina y el Caribe: diagnóstico sobre la situación y las políticas. Santiago de Chile: CEPAL, 2003: 8-4I.

6. Hijar M, Arredondo A, Carrillo C, Solórzano L. Road traffic injuries in an urban area in Mexico. An epidemiological and cost analysis. Accid Anal Prev 2004; 36:37-42.

7.Aparicio R.Transición demográfica y vulnerabilidad durante la vejez. En: La situación demográfica de México 2002. México: CONAPO, 2003:I55-167.

8. Hernández FD. Hogares, pobreza y vejez. Desigualdad y pobreza de la población mayor. Demos 200I; 14:32-33.

9. Ministerio de Trabajo y Asuntos Sociales de España, II Asamblea Mundial de Envejecimiento. España: Ministerio de Trabajo y Asuntos Sociales, 2002.

10.Zúñiga E, Gómez C. Pobreza, curso de vida y envejecimiento poblacional en México. En:La situación demográfica de México 2002. México:CONAPO, 2003:| $14|-| 53$.

II. Instituto Nacional de Estadística, Geográfica e Informática. Estadísticas a propósito del día internacional de las personas de edad. México: INEGI, 2003 y 2005.

12. Instituto Nacional de Estadística, Geográfica e Informática. Estadísticas demográficas 2003. Cuaderno 20. México: INEGI, 2004.

13. Instituto Nacional de Estadística, Geográfica e Informática. Estadísticas demográficas 2004. Cuaderno 16. México: INEGI, 2005.

14. Estadística de egresos hospitalarios del sector público del Sistema $\mathrm{Na}$ cional de Salud, 2003. Salud Publica Mex 2004; 46(5):464-487.

15. Marín I P. Fragilidad en el adulto mayor y valoración geriátrica integral. Rev Med Chil 2000;29:1-2.

16. Consejo Nacional de Población. Estadísticas índice y grado de marginación por localidad 2000. México: CONAPO, 200I.

17. Folstein MF, Folstein SE, McHugh PR.'Mini-Mental State':a practical method for grading the cognitive state of patients for the clinician.J of Psychiatr Res 1975; 12: 189-198. 
18. Salgado-de SnyderVN,Wong R. Envejeciendo en la pobreza: género, salud y calidad de vida. México. México: Instituto Nacional de Salud Pública, 2003. 19. Radloff LS. The CES-D scale:A self-report depresión scale for research in the general population. Applied Psychological Measurement 1977; I: 385-401.

20. Hosmer DW, Lemeshow S.Applied logistic regression. Jonh Wiley \& Sons. New York: Interscience Publication, 2000.

21. Ruelas-González MG, Salgado-de Snyder VN. El maltrato en el adulto mayor: factores de riesgo en un contexto de pobreza. En:Salgado-de Snynder VM,Wong R. Envejecimiento, pobreza y salud en población urbana. México: Instituto Nacional de Salud Pública, 2006.

22. Olaiz G, Rojas R, Barquera S, Shamah T,Aguilar C, Cravioto P et al. Encuesta Nacional de Salud 2000. México: Instituto Nacional de Salud Pública, 2003. 23. Arlaes-Yero L. Factores de riesgo asociados a los accidentes en el hogar. Rev Gen Integr 1998; I4(6):58I-585

24. Organización Mundial de la Salud. Evaluación farmacológica del adulto mayor. Cuaderno 6. Nueva York: OMS, 2002.

25. Rozenfeld S, Camacho LA,Veras P. Medication as a risk factor for falls in older women in Brazil. Rev Panam Salud Publica 2003;13(6):369-375.

26. Miñana-Climent JC. Características y factores asociados a las caídas en pacientes con enfermedad cerebrovascular. Rev Esp Geriatr Gerontol 2005;40:2.

27. Marín-Larraín P. Manual de geriatría y gerontología. Santiago de Chile: Universidad Católica de Chile, 2002.

28. Molina Y]. Caídas en el adulto mayor. Compendio de geriatría clínica. Santiago de Chile: Universidad Católica de Chile, 2000.

29. Solis L, Velez A, Berumen D. Prevalencia del consumo de bebidas alcohólicas y problemas asociados en personas de la tercera edad que acuden a los servicios de atención primaria de la ciudad de México. Salud Mental 2000; $23: 3$.

30. Celis A, Valencia N. Traumatismos y envenenamientos en Jalisco. Salud Publica Mex 1991;33:77-87.

3I. Kevin RH.Alcohol abuse and dependence: a clinical update on alcoholism in the older population. Geriatrics 2004;59:9.

32. Baro F. Factores psicosociales y la salud de los ancianos. En: Hacia el bienestar de los ancianos. Nueva York: OPS, 2002.

33. Roberto KA. The role of social supports in older women's recovery from hip fractures. J Appl Gerontol 1992; I (3):3 I4-325.

34. Cummings SR, Phillips SL, Wheat ME, Black D, Goosby E. Recovery of function after hip fracture. The role of social supports. J Am Geriatr Soc 1998;36(9):801-806.

35. Ávila-Burgos L, Ramirez-Valverde G, Martínez-Damian MA, Cruz-Valdez A, Santiago-Cruz MJ, Medina-Solis CE. Socioeconomic determinants of inequality and self-reportes morbidity among adolescents in a developing country. Saudi Med J 2005;26(10).

36. Wong R, Pelaez M, Palloni A. Autoinforme de salud general en adultos mayores de América Latina y el Caribe: su utilidad como indicador. Rev Panam Salud Publica 2005; 17:5-6.

37. Alustiza-Galarza A. Entorno familiar de las personas con discapacidad. Algunos resultados preliminares para el país Vasco de la encuesta sobre discapacidades, deficiencias y estado de salud. Rev Esp Geriatr Gerontol 2005;40:06.

38. Gutiérrez-Robledo LM, García-Mayo EJ. Salud y envejecimiento de la población en México. México: ONU, 2005. 